\title{
Deadenylation of maternal mRNAs during Xenopus oocyte maturation does not require specific cis-sequences: a default mechanism for translational control
}

\author{
Susan M. Varnum ${ }^{1}$ and W. Michael Wormington ${ }^{2,3}$ \\ ${ }^{1}$ Department of Biology, Brandeis University, Waltham, Massachusetts 02254 USA; ${ }^{2}$ Department of Biology, University of \\ Virginia, Charlottesville, Virginia 22901 USA
}

\begin{abstract}
The meiotic maturation of Xenopus oocytes initiates significant changes in the translation of a number of maternal mRNAs that coincide with alterations in their polyadenylation states. A considerable number of maternal mRNAs are deadenylated in mature oocytes, thereby reducing their translational efficiencies. In this report we demonstrate that deadenylation does not require specific cis-acting sequences. Polyadenylated RNAs derived from either ribosomal protein or $\beta$-globin mRNAs, or that contain non-mRNA-derived sequences, are deadenylated in mature oocytes. Translation of a substrate RNA is not required for its deadenylation. G10 mRNA is representative of a class of mRNAs that is translationally activated at maturation and contains the cytoplasmic polyadenylation element (U) ${ }_{6} \mathrm{AU}$. A deletion mutant $\mathrm{G} 10$ transcript that lacks the $(\mathrm{U})_{6} \mathrm{AU}$ element is not polyadenylated in mature oocytes but is deadenylated instead. Insertion of the (U) ${ }_{6} \mathrm{AU}$ element into the 3 '-untranslated region of the ribosomal protein L1 mRNA is sufficient to prevent both its deadenylation and polysomal release in mature oocytes. These results indicate that the deadenylation and translational inactivation of maternal mRNAs during Xenopus oocyte maturation occur by a default pathway in which transcripts lacking a cytoplasmic polyadenylation element undergo poly(A) removal.
\end{abstract}

[Key Words: Poly(A) removal; maternal mRNAs; Xenopus oocytes; meiosis; translational control]

Received August 22, 1990; revised version accepted October 11, 1990.

A large pool of maternal mRNAs is synthesized and sequestered during Xenopus oogenesis. The stage-specific post-transcriptional and -translational regulation of these stored mRNAs determines all protein synthesis between the meiotic maturation of fully grown oocytes and development of the 4000-cell blastula embryo when zygotic transcription is initiated (for review, see Davidson 1986). The induction of oocyte maturation in several organisms initiates significant changes in the translational utilization of a number of maternal mRNAs that coincide with alterations in their polyadenylation states /for reviews, see Jackson and Standart 1990; Wickens 1990).

In Xenopus, a considerable number of maternal mRNAs are deadenylated in mature oocytes, thereby reducing the total poly(A) content by $\sim 40 \%$ (Sagata et al. 1980 . In general, this deadenylation correlates with markedly reduced translational efficiencies, as observed for the mRNAs encoding cytoskeletal actin /Sturgess et al. 1980), ribosomal proteins (Hyman and Wormington

${ }^{3}$ Corresponding author.
1988 ), and translation elongation factor $1 \alpha$ (Wormington 1989|. The core histone mRNAs are an exception to this correlation because their translation increases significantly after maturation despite their deadenylation ( $\mathrm{Ru}$ derman et al. 1979). Conversely, the translational activation of maternal mRNAs such as D7, G10, and c-mos in mature oocytes correlates with their cytoplasmic polyadenylation (Dworkin and Dworkin-Rastl 1985; Dworkin et al. 1985; Sagata et al. 1988; Fox et al. 1989; McGrew et al. 1989). The translational activity of a third class of maternal mRNAs that includes histone $\mathrm{Hl}$ (Woodland et al. 1979), fibronectin (Lee et al. 1984), lamin (Stick and Hausen 1985), and c-myc (Taylor et al. $1986)$ is not altered during maturation.

The cis-acting sequence requirements for maturationspecific changes in the polyadenylation and translation of maternal mRNAs have been investigated by the microinjection of synthetic RNAs into Xenopus oocytes. The cytoplasmic polyadenylation and translational activation of synthetic D7 and G10 mRNAs during oocyte maturation require two discrete cis-acting elements, a $\mathrm{U}$-rich sequence |referred to hereafter as the cytoplasmic 
polyadenylation element (CPE)], and the ubiquitous nuclear polyadenylation signal (AAUAAA), which are present in the $3^{\prime}$-untranslated regions of these mRNAs (Fox et al. 1989; McGrew et al. 1989; McGrew and Richter 1990|. These sequences are sufficient to confer maturation-specific polyadenylation and increased translation when fused to a Xenopus $\beta$-globin mRNA that is otherwise not subject to this regulation (McGrew et al. 1989/. In the case of G10 mRNA, it appears that the actual process of polyadenylation and not the presence of a poly(A) tail per se is required for its translational activation (McGrew et al. 1989). U-rich sequences that closely resemble the CPEs found in G10 and D7 mRNAs are also present in the $3^{\prime}$-untranslated regions of additional transcripts that are polyadenylated during oocyte maturation in Xenopus as well as in other organisms (Fox et al. 1989; McGrew et al. 1989; McGrew and Richter 1990; Paris and Richter 1990).

Initial studies from our laboratory showed that a synthetic ribosomal protein (r-protein) Ll mRNA, when microinjected into stage VI oocytes, is deadenylated and released from polysomes simultaneously with the translational inactivation of endogenous r-protein mRNAs upon maturation (Hyman and Wormington 1988). These studies also indicated that sequences located within 387 nucleotides of the $3^{\prime}$ end of the L1 mRNA direct maturation-specific deadenylation and polysomal release when fused to Xenopus $\beta$-globin mRNA. In this report we have extended these studies to address the sequence specificity of deadenylation. We demonstrate that deadenylation does not require specific cis-acting sequences. Polyadenylated RNAs that are derived from either $r$-protein or $\beta$-globin mRNAs or that contain a homopolymer sequence are deadenylated in mature oocytes. A deletion mutant G10 mRNA that lacks the CPE, $(\mathrm{U})_{6} \mathrm{AU}$, is neither polyadenylated nor associated with polysomes in mature oocytes but is deadenylated. Insertion of the $(U)_{6} \mathrm{AU}$ element into the $3^{\prime}$-untranslated region of the $r$-protein L1 mRNA is sufficient to prevent both its deadenylation and polysomal release in mature oocytes. These results indicate that the deadenylation and translational inactivation of maternal mRNAs during Xenopus oocyte maturation occur by a default pathway in which transcripts lacking a CPE undergo poly|A) removal.

\section{Results \\ Deadenylation and polysomal release of a synthetic $r$ - protein S22 mRNA in mature oocytes}

Our initial studies showed that an SP6-derived L1 mRNA was deadenylated and released from polysomes after the maturation of microinjected oocytes, thereby mimicking the translational inactivation of endogenous r-protein mRNAs (Hyman and Wormington 1988). To extend these analyses, the regulation of an SP6-derived $r$-protein S22 mRNA was also characterized in microinjected oocytes (Fig. 1A). The polyadenylation state and translation of the SP6-derived S22 mRNA were determined before and after progesterone-induced maturation
A
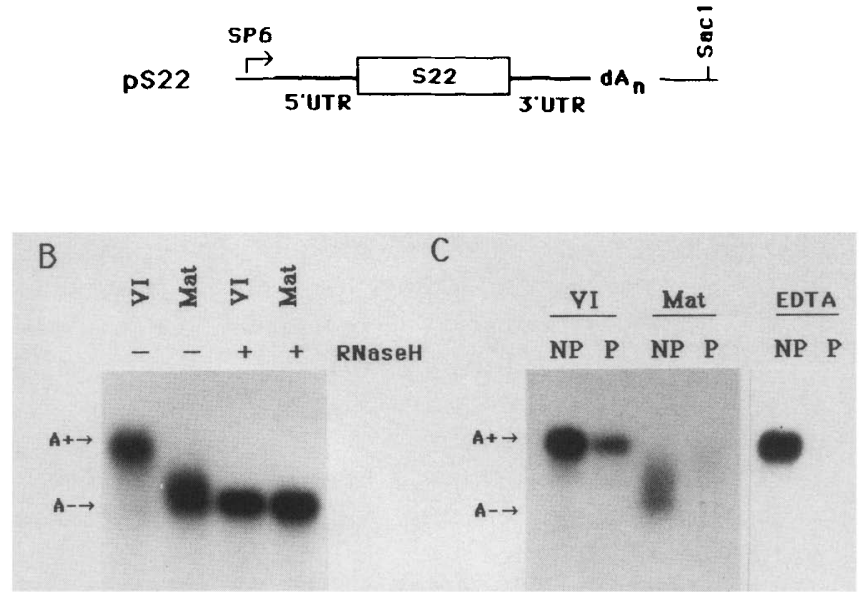

Figure 1. Deadenylation and polysome release of S22 mRNA. (A) Schematic of pS22 that contains a full-length S22 cDNA. Radiolabeled SP6-derived S22 mRNA with a poly $(\mathrm{A})_{-120}$ tail was synthesized from a template linearized with SacI and 3'protruding ends removed by T4 DNA polymerase. SP6 RNA was microinjected into the cytoplasm of stage VI oocytes that were incubated in either the presence or absence of $1 \mu \mathrm{g} / \mathrm{ml}$ of progesterone. $(B)$ Total RNA was isolated from stage VI (VI lanes) and mature oocytes (Mat lanes). In some cases, total RNA was hybridized to oligo(dT) and incubated with RNase $\mathrm{H}$ |+ RNase $H$ lanes $\mid$. RNAs were electrophoresed on a $4 \%$ polyacrylamide-urea gel. $(C)$ Polysomal $(\mathrm{P})$ and nonpolysomal $(\mathrm{NP})$ RNA was isolated from stage VI and mature oocytes and electrophoresed on a $1.3 \%$ agarose-formaldehyde gel. Treatment of the stage VI oocyte cytoplasmic extract with EDTA prior to centrifugation through the $20 \%$ sucrose cushion resulted in the complete release of S22 mRNA from the polysomal pellet (P) into the nonpolysomal (NP) supernatant.

in vitro. Figure $1 \mathrm{~B}$ shows that the microinjected SP6-derived S22 transcript was reduced in length after oocyte maturation. To confirm that this transcript was deadenylated, total RNA was extracted from microinjected stage VI and mature oocytes, hybridized to oligo(dT), and digested with RNase $\mathrm{H}$ (Fig. 1B). In vitro deadenylation with RNase $\mathrm{H}$ reduced the length of SP6-derived S22 mRNA recovered from stage VI oocytes but did not alter the size of this transcript isolated from mature oocytes. The SP6-derived S22 mRNA isolated from mature oocytes was virtually identical in length to that recovered from stage VI oocyte RNA digested with RNase $\mathrm{H}$, indicating that the poly(A) tail was removed from the S22 transcript after maturation.

RNA was prepared from polysomal (P) and nonpoly. somal (NP) fractions isolated from microinjected oocytes. Figure $1 \mathrm{C}$ shows that while the polyadenylated SP6-derived S22 mRNA was translated in stage VI oocytes, the deadenylated transcript in mature oocytes was not associated with polysomes. Thus, the SP6 S22 mRNA is deadenylated and translationally inactivated in mature oocytes, as observed previously for other $r$ protein mRNAs (Baum and Wormington 1985; Hyman and Wormington 1988). 
Xenopus $\beta$-globin mRNA is deadenylated and released from polysomes in mature oocytes

Our initial analyses of the translational inactivation of the SP6 L1 mRNA in mature oocytes also utilized the plasmid pSP64-X $\beta \mathrm{m}$ to synthesize Xenopus $\beta$-globin mRNA (Hyman and Wormington 1988). This transcript was chosen as a control because previous studies had indicated that the translational efficiency of rabbit globin mRNA in microinjected oocytes increased in parallel with the twofold stimulation in overall protein synthesis after the induction of maturation (Richter et al. 1982). Our results were consistent with this observation and showed that the pSP64-X $\beta \mathrm{m}$-derived mRNA was not deadenylated and remained polysomal in mature oocytes (Hyman and Wormington 1988). However, the pSP64-X $\beta \mathrm{m}$ transcript contains only a $3^{\prime}$ poly $(\mathrm{A})_{23}$ tract followed by a poly $\mid \mathrm{C})_{30}$ tail (Krieg and Melton 1984). Thus, the failure of this transcript to be deadenylated could be due to either its short poly(A) tract or the presence of the extraneous poly $(\mathrm{C})$ tail as opposed to the absence of specific cis-acting sequences required for poly(A) removal. To address these possibilities, we determined the minimal poly $(\mathrm{A})$ tail length required for deadenylation and have examined the regulation of a $\beta$ globin mRNA that contains a $3^{\prime}$ poly $(\mathrm{A})_{50-100}$ tail and lacks the extraneous poly $(C)$ sequences encoded by the pSP64-X $\beta \mathrm{m}$ template.

To ascertain the minimal poly $|\mathrm{A}|$ tail length requirement for deadenylation, we synthesized SP6 L1 mRNAs that contained $3^{\prime}$ poly $(\mathrm{A})$ tails of either 20 or 42 adenylate residues in length (Fig. 2A). Figure $2 \mathrm{~B}$ shows that while the poly $|\mathrm{A}|_{20}$ tail was not removed from L1-20 RNA in mature oocytes, the Ll-42 RNA with a poly $(\mathrm{A})_{42}$ tail, was deadenylated. Both transcripts were substrates for deadenylation in vitro by $\mathrm{RNase} \mathrm{H}$ after hybridization to oligo(dT). Therefore, a poly $(\mathrm{A})_{20}$ tail is insufficient for deadenylation in mature oocytes.

These results suggest that the poly $(\mathrm{A})_{23}$ tract on the pSP64-X $\beta \mathrm{m}$ transcript is probably insufficient in length to be removed in mature oocytes. Therefore, we removed both the poly $(\mathrm{A})$ and poly $(\mathrm{C})$ homopolymer tracts from this SP6 RNA by hybridization to oligo(dT) and digestion with RNase $\mathrm{H}$ (Fig. 3A). A 3' poly $(\mathrm{A})_{50-100}$ tail was then added with Escherichia coli poly|A) polymerase. This modified transcript, which more closely resembles native Xenopus $\beta$-globin mRNA, was deadenylated and released from polysomes in mature oocytes (Fig. 3B,C). We have not resolved the apparent discrepancy between this result and the elevated translation of rabbit globin mRNA in mature oocytes reported initially by Richter et al. (1982). We note, however, that Richter et al. (1982) microinjected 20-40 ng of partially purified rabbit globin mRNA, whereas we have used l ng of synthetic Xenopus $\beta$-globin mRNA. We conclude, therefore, that Xenopus $\beta$-globin mRNA does not require sequences derived from the r-protein L1 mRNA to specify its deadenylation and translational inactivation in mature oocytes as proposed originally (Hyman and Wormington 1988).
A
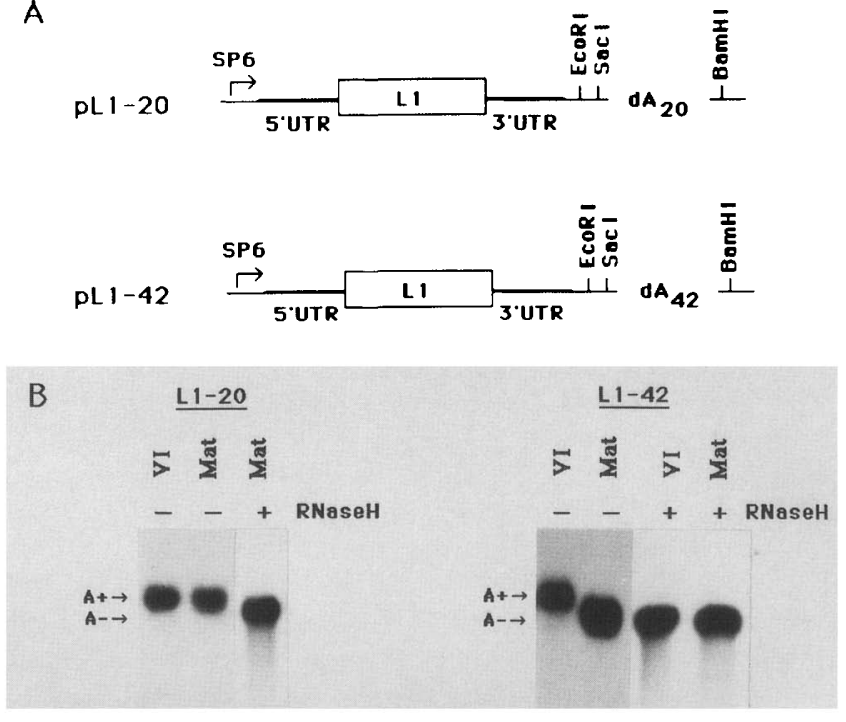

Figure 2. Poly $|\mathrm{A}|$ tail length requirement for deadenylation. (A) Diagram of the SP6-Ll plasmid DNAs that were linearized with $B a m H I$ for use as transcription templates. $|B|$ Radiolabeled SP6 L1-20 and L1-42 RNAs with poly $\mid \mathrm{A})_{20}$ and poly $(\mathrm{A})_{42}$ tails, respectively, were microinjected into stage VI oocytes that were incubated in either the presence or absence of progesterone. Total RNA was extracted from stage VI and mature oocytes. In some cases, total RNA was hybridized to oligo|dT) and incubated with RNase $\mathrm{H}$ ( + RNase $\mathrm{H}$ lanes). RNAs were electrophoresed on a $1 \%$ agarose-formaldehyde gel.

\section{Deadenylation does not require specific cis-acting sequences}

The deadenylation of Xenopus $\beta$-globin mRNA raised the possibility that the removal of poly $(\mathrm{A})$ from endogenous oocyte mRNAs may also not require specific cisacting sequences. To address this issue, transcripts derived from the 3'-untranslated region (UTR) of the S22 mRNA were constructed and analyzed for maturationspecific deadenylation in microinjected oocytes (Fig. 4A). The wild-type S22 3'-UTR RNA was deadenylated in mature oocytes (Fig. 4B). Two mutant templates were constructed that altered a total of 34 of the 45 nucleotides present in the S22 3' UTR (Fig. 4A). In S22-mut2 RNA, 14 nucleotides were altered, including the canonical poly $|\mathrm{A}|$ addition site, AAUAAA, and the sequence, UUCUGUUU, that is conserved among Xenopus r-protein mRNAs (Mariottini et al. 1988). In S22-mut3 RNA, the 3 '-terminal 20 nucleotides, including a second sequence, GUGGAUUG, which is also conserved among Xenopus r-protein mRNAs (Mariottini et al. 1988), were altered. Figure $4 \mathrm{~B}$ shows that both mutant RNAs were deadenylated in mature oocytes. A smaller proportion of S22-mut2 RNA was deadenylated relative to the wildtype transcript, suggesting that specific sequences may influence the efficiency of poly(A) removal. Both mutant S22 RNAs were deadenylated more extensively in mature oocytes than by treatment with RNase $\mathrm{H}$ after hybridization to oligo(dT). Thus, neither the poly $(\mathrm{A})$ addition site nor the two elements conserved in Xenopus rprotein mRNAs are required for maturation-specific 
A
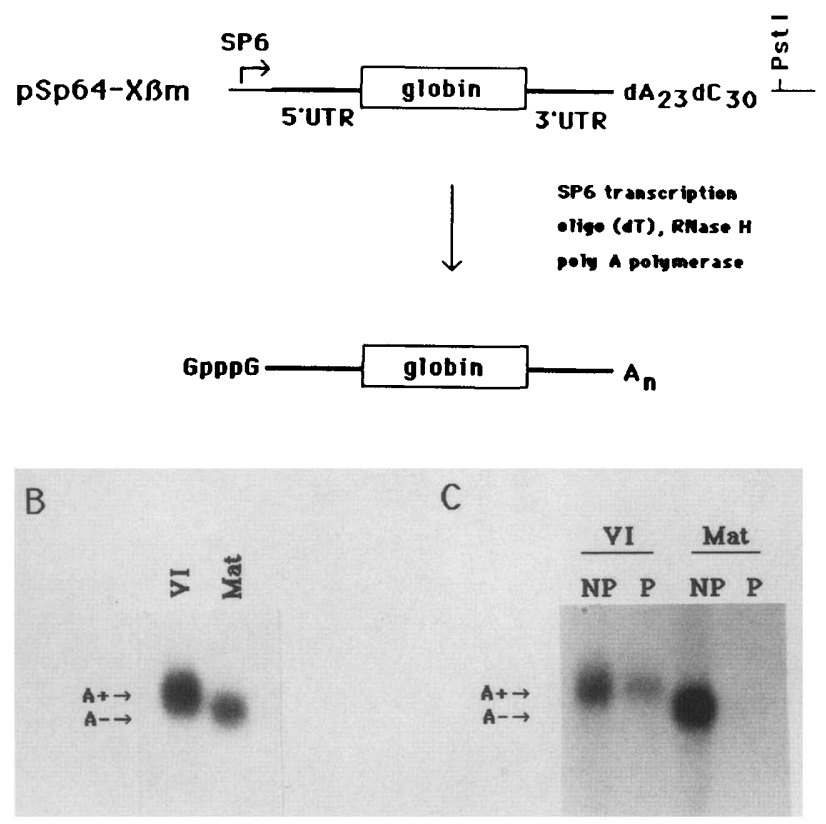

Figure 3. Globin mRNA is deadenylated and released from polysomes in mature oocytes. $(A)$ Diagram of the pSP64-X $\beta \mathrm{m}$ transcription plasmid. Radiolabeled SP6 X $\beta m$ RNA was synthesized from a template linearized with PstI, and 3 '-protruding ends were removed by T4 DNA polymerase. The RNA was hybridized to oligo(dT) and digested with RNase $\mathrm{H}$, and a poly $(A)_{50-100}$ tail was added with poly(A) polymerase. SP6 RNA was microinjected into stage VI oocytes that were incubated in either the presence or absence of progesterone. $|B|$ Total RNA was extracted from stage VI and mature oocytes. (C) RNA extracted from nonpolysomal $(\mathrm{NP})$ and polysomal $|\mathrm{P}|$ fractions of stage VI and mature oocytes. RNAs were electrophoresed on a $1.3 \%$ agarose-formaldehyde gel.

deadenylation.

As an additional test for the absence of sequence specificity for deadenylation, we constructed a transcription template in which a homopolymer $\left[\left.(\mathrm{dC}) \cdot(\mathrm{dG})\right|_{24}\right.$ oligonucleotide, flanked by initiation and termination codons, was inserted adjacent to the $\left[(\mathrm{dA}) \cdot\left\langle\mathrm{dT} \|_{100}\right.\right.$ tract in pSP65AT (Fig. 5A). Figure 5B shows that the HP1 RNA was deadenylated in mature oocytes, providing additional evidence that maturation-specific deadenylation does not require specific cis-acting sequences.

\section{Deadenylation does not require translation of a substrate RNA}

To determine whether a substrate RNA must be associated with polysomes for its deadenylation, we synthesized a polyadenylated RNA fragment, G52, that contains the terminal 52 nucleotides from the 3 '-untranslated region of Xenopus $\beta$-globin mRNA (Fig. $5 \mathrm{C}$ ). The G52 transcript lacks a consensus translation initiation sequence and contains no internal AUGs. This RNA was not associated with polysomes in stage VI oocytes (Fig. 5D). The exclusion of G52 RNA from the polysomal pellet was not due to its short length because the similarly sized wild-type S22 3' UTR RNA, which con- tains a consensus translation initiation sequence, can be efficiently recovered in the polysomal pellet (S.M. Varnum, unpubl.). Figure 5D shows that the nontranslatable G52 RNA was deadenylated following maturation. This result is consistent with our initial observation that the nonpolysomal fraction of endogenous $r$-protein mRNAs is deadenylated in mature oocytes (Hyman and Wormington 1988).

\section{The cytoplasmic polyadenylation element $(U)_{6} A U$ prevents deadenylation}

A class of maternal transcripts that includes the G10 and D7 mRNAs escapes deadenylation in mature oocytes (Fox et al. 1989; McGrew et al. 1989; Paris and Richter 1990). The cytoplasmic polyadenylation of these transcripts may prevent their deadenylation. In contrast
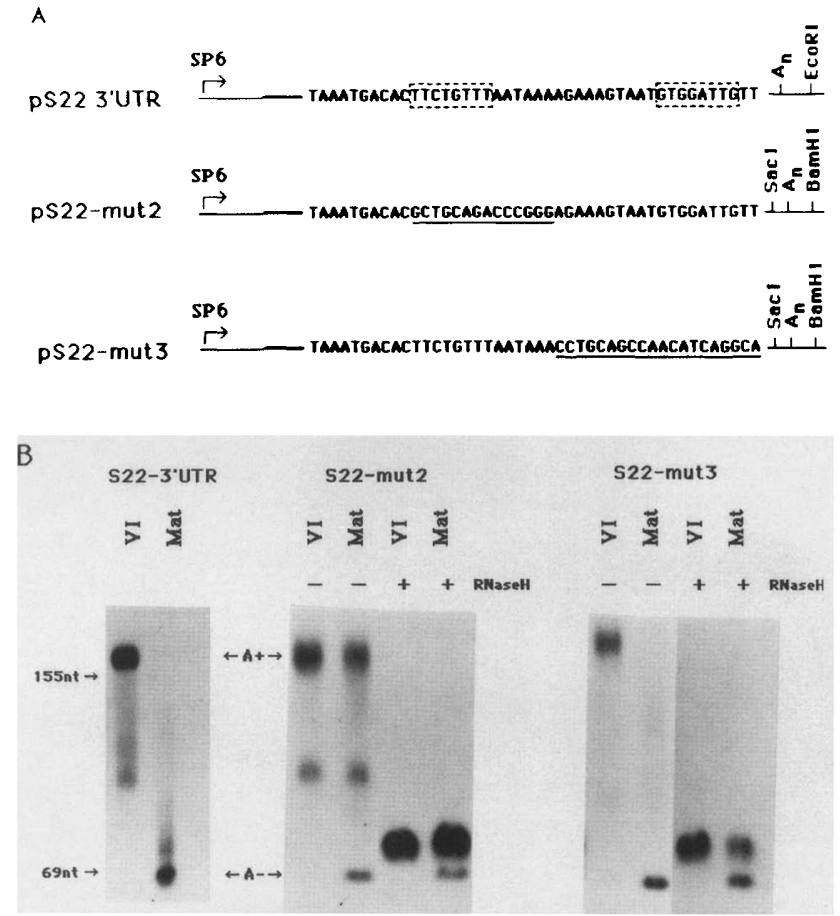

Figure 4. Deadenylation of wild-type and mutant transcripts containing the S22 3' UTR. (A) Schematic of the wild-type and mutant pS22 3' UTR transcription plasmids. The hatched boxes indicate the $3^{\prime}$-untranslated consensus regions 3 and 4 that are conserved among Xenopus r-protein mRNAs. The underlined sequences indicate the regions mutated in pS22-mut2 and pS22-mut3. Radiolabeled SP6 RNAs with 3' poly $(\mathrm{A})_{100}$ tails were synthesized from templates linearized with EcoRI (pS223'UTR) or BamHI (pS22-mut2 and pS22-mut3). (B) SP6 RNAs were microinjected into stage VI oocytes that were incubated in either the presence or absence of progesterone. Total RNA was extracted from stage VI and mature oocytes and electrophoresed on a $6 \%$ polyacrylamide-urea gel. Some of the RNA isolated from oocytes microinjected with S22-mut 2 and S22-mut3 RNAs was hybridized to oligo(dT) and incubated with RNase $\mathrm{H}$ prior to electrophoresis (+ RNase $\mathrm{H}$ lanes). The labeled transcript migrating between the polyadenylated and deadenylated S22-mut2 RNAs was an irrelevant contaminant of unknown origin present in the initial RNA preparation. 
to deadenylation, cytoplasmic polyadenylation requires a CPE and the nuclear polyadenylation signal AAUAAA (Fox et al. 1989; McGrew et al. 1989). The AAUAAA element is insufficient to confer resistance to poly $(\mathrm{A})$ removal because this sequence is also present in mRNAs that are deadenylated in mature oocytes. To determine whether the CPE, $(\mathrm{U})_{6} \mathrm{AU}$, prevents deadenylation, we analyzed the regulation of $\beta$-globin-G10 fusion RNAs whose substrate activities for polyadenylation have been characterized previously (Fig. 6A; McGrew et al. 1989). Extraneous vector sequences that prevent cytoplasmic polyadenylation (McGrew et al. 1989) were removed from the SP6 RNAs by hybridization to oligo(dT) and digestion with RNase $\mathrm{H}$. Figure $6 \mathrm{~B}$ shows that the fusion transcript XBG10 $\Delta 763-797 / \mathrm{dT}$ underwent maturationspecific polyadenylation in microinjected oocytes. In contrast, the XBG10 $0741-748 / \mathrm{dT}$ transcript lacking the $(\mathrm{U})_{6} \mathrm{AU} \mathrm{CPE}$ was not polyadenylated in mature oocytes (Fig. 6C). To determine whether this mutant transcript was deadenylated, a 3' poly $(\mathrm{A})_{\sim 100}$ tail was added posttranscriptionally with $E$. coli poly(A) polymerase. As shown in Figure 6D, the poly(A) tail on the XBG10د741$748 / \mathrm{dT} / \mathrm{A}^{+}$RNA was not elongated in mature oocytes, but was deadenylated instead.

These results suggest that the deadenylation of maternal mRNAs could occur by default in the absence of CPE-dependent cytoplasmic polyadenylation. To address this potential mechanism further, we determined whether insertion of the $(\mathrm{U})_{6} \mathrm{AU} \mathrm{CPE}$ into the $3^{\prime}$-untranslated region of the r-protein Ll mRNA was sufficient to prevent its deadenylation and translational inactivation. The $(\mathrm{U})_{6} \mathrm{AU}$ sequence was inserted into the the Ll mRNA at the same position relative to the poly $(A)$ addition site as it is found in the G10 mRNA
(Fig. 7A). Extraneous vector sequences were removed from the resultant $\mathrm{Ll}-1095$ RNA, and a poly $(\mathrm{A})_{\sim 100}$ tail was added with $E$. coli poly $(\mathrm{A})$ polymerase. Figure $7 \mathrm{~B}$ shows that the poly $(A)_{100}$ tail on the $\mathrm{L} 1 \mathrm{mRNA}$ was removed in mature oocytes. In contrast, the presence of the $(\mathrm{U})_{6} \mathrm{AU}$ element in L1-1095 RNA resulted in the maturation-specific extension of its poly(A) tail (Fig. 7B). Additional experiments indicated that a nonadenylated L1-1095 RNA injected into oocytes was also polyadenylated at maturation analogous to the behavior of the XBG10 $1763-797 /$ dT transcript (Fig. 7B; data not shown). The translational efficiency of the L1 mRNA in mature oocytes was determined by the fate of its poly(A) tail. The wild-type $\mathrm{Ll}$ mRNA was released from polysomes concomitant with its deadenylation in mature oocytes (Fig. 7B). However, the L1-1095 transcript not only retained a poly(A) tail after maturation but also remained associated with polysomes. Thus, the CPE-dependent persistence of a poly $|A|$ tail is sufficient to prevent the translational inactivation of a r-protein mRNA in mature oocytes.

\section{Discussion}

A default pathway for the deadenylation and translational inactivation of maternal mRNAs during oocyte maturation

A translational control mechanism is established during Xenopus oocyte maturation that discriminates between two classes of maternal mRNAs. One class of mRNAs undergoes a sequence-dependent cytoplasmic polyade-
Figure 5. An RNA containing a poly $(\mathrm{C})_{24}$ homopolymer tract and a nontranslatable RNA are deadenylated in mature oocytes. (A) Diagram of the pHPl transcription plasmid. Radiolabeled SP6 HP1 RNA with a $3^{\prime} \operatorname{poly}(A)_{100}$ tail was synthesized from a template linearized with BamHI. (B) SP6 HPl RNA was microinjected into stage VI oocytes that were incubated in either the presence or absence of progesterone. Total RNA was extracted from stage VI and mature oocytes and electrophoresed on a $6 \%$ polyacrylamide-urea gel. (C) Schematic of the pG52 transcription plasmid that contains the final 52 bp of the SP64-X $\beta$ m 3' UTR. Radiolabeled SP6 G52 RNA with a 3' poly $(A)_{100}$ tail was synthesized from a template linearized with BamHI. SP6 RNA was microinjected stage VI oocytes that were incubated in either the presence or absence of progesterone. $(D)$ RNA extracted from nonpolysomal (NP) and polysomal (P) fractions of stage VI and mature oocytes was electrophoresed on a $6 \%$ polyacrylamide-urea gel.
A pHP 1
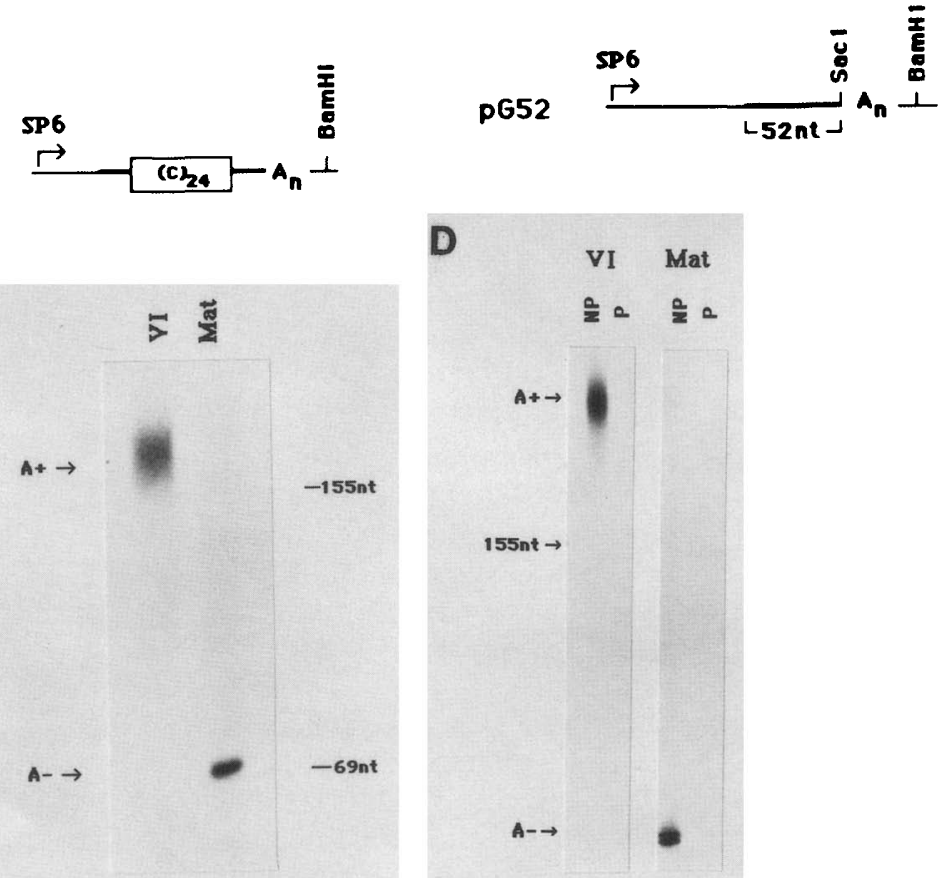
A

PXBG 10

pXBG 10 $0763-797$

pXBG 10 $0741-748$

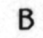

B

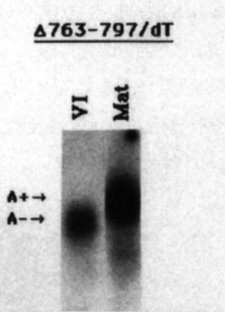

C $\Delta 741-748 / d T$ $5:$

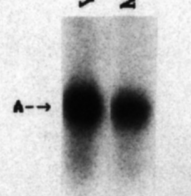

D

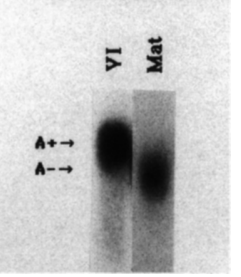

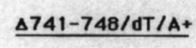

Figure 6. Changes in poly $(A)$ tail lengths of wild-type and deletion mutant $\beta$-globin/G10 fusion RNAs. $(A)$ Schematic of XBG10 plasmids that contain wild-type or deletion mutant $G 10$ 3' UTR fused to Xenopus $\beta$-globin-coding sequences. The cytoplasmic polyadenylation sequences TTTTTTAT and AATAAA are indicated. Deleted sequences are denoted with parentheses. Radiolabeled SP6 RNAs were synthesized from templates linearized with SalI. SP6 XBG10 $163-797$ and XBG10د741-748 RNAs were hybridized to oligo(dT) and digested with RNase

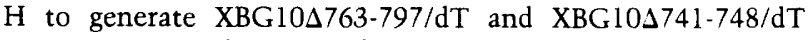
RNAs, respectively. Some of the XBG10 $\Delta 741-748 / \mathrm{dT}$ RNA was treated with poly(A) polymerase to produce XBG10 $1741-748$ / $\mathrm{dT} / \mathrm{A}^{+}$RNA that contains a poly $|\mathrm{A}|_{100}$ tail. $(B-D)$ SP6 RNAs were microinjected into stage VI oocytes that were incubated in either the presence or absence of progesterone. Total RNA was extracted from stage VI and mature oocytes and electrophoresed on a $1.3 \%$ agarose-formaldehyde gel.

nylation and recruitment onto polysomes (Fox et al. 1989; McGrew et al. 1989|. The second class represents a substantial number of maternal mRNAs that are deadenylated and released from polysomes in mature oocytes (Dworkin and Dworkin-Rastl 1985; Dworkin et al. 1985; Hyman and Wormington 1988). We have shown that poly $(\mathrm{A})$ removal does not require specific cis-acting sequences but instead is the default fate of mRNAs that lack a CPE.

We proposed previously that cis-acting sequences are involved in specifying deadenylation (Hyman and Wormington 1988/. This proposal was based on the observation that sequences located near the 3 ' end of the r-protein Ll mRNA directed maturation-specific deadenylation when fused to a Xenopus $\beta$-globin mRNA that was otherwise not subject to this regulation. In this report we have shown that a Xenopus $\beta$-globin mRNA, with an appropriate $3^{\prime}$ terminus, is indeed deadenylated. In addition, extensively mutated transcripts derived from the 3 '-untranslated region of the r-protein S22 mRNA were all deadenylated in mature oocytes. The reduced amount of deadenylated S22-mut2 RNA suggests, however, that specific sequences may influence the efficiency of poly(A) removal. Nonetheless, the deadenylation of a homopolymer RNA provides the strongest evidence that this RNA processing event does not require specific sequences.

Deadenylation does require a minimal poly(A) tract length. Although a poly $(\mathrm{A})_{42}$ tail can be removed at maturation, a transcript containing only a poly $(\mathrm{A})_{20}$ tract is not deadenylated. This probably explains why a $\beta$-globin mRNA with a poly $(A)_{23}$ tail was not deadenylated in our previous studies (Hyman and Wormington 1988). It is also possible that the presence of an extraneous poly $(\mathrm{C})_{30}$ tail inhibited the deadenylation of this transcript. However, deadenylation does not require that the poly $(\mathrm{A})$ tail must actually terminate with an adenylate residue. The 3 ' ends of several SP6-derived RNAs used in previous studies (Hyman and Wormington 1988) and in this report are terminated by 3 to 5 nonadenylate residues of polylinker sequence immediately distal to their poly(A) tracts. Nonetheless, these RNAs are deadenylated.

The nontranslatable G52 RNA was deadenylated at maturation, demonstrating that mRNAs do not need to be associated with polysomes to undergo poly(A) removal. This indicates that nontranslating mRNAs are not required to cycle through polysomes for their deadenylation at maturation. This absence of a translation requirement differs from the regulation of tubulin and histone mRNA turnover in mammalian cells in which only polysomal transcripts are targeted for degradation (for reviews, see Cleveland 1988; Marzluff and Pandey 1988). The deadenylation of nonpolysomal mRNAs in mature oocytes suggests that this activity may differ

A

pLI

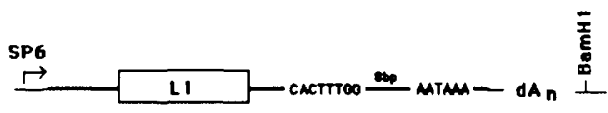

pl $1-1095$
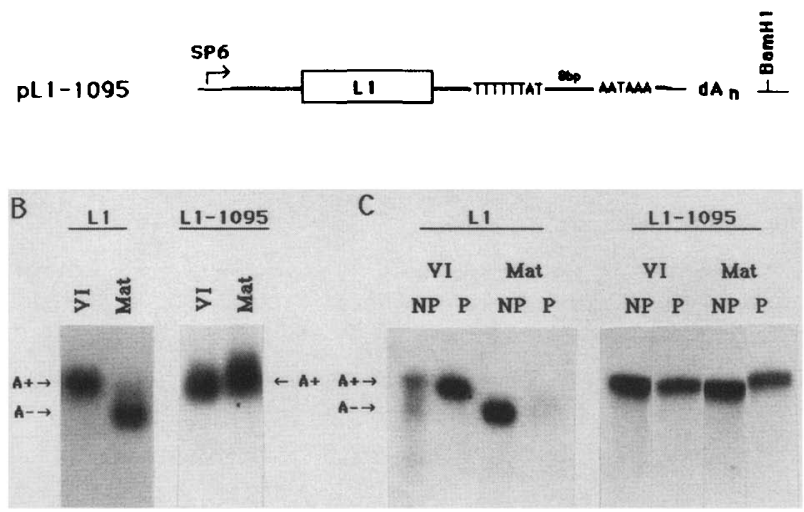

Figure 7. The $(\mathrm{U})_{6} \mathrm{AU}$ element prevents the deadenylation of L1 mRNA. $(A)$ Diagram of the wild-type pLl plasmid and the pL1-1095 plasmid that contains the G10-derived sequence TTTTTTAT, inserted 8 bp upstream of the AATAAA element located in the 3 '-untranslated region of the $L 1$ mRNA. Radiolabeled SP6 RNAs with $3^{\prime}$ poly $(A)_{100}$ tails were synthesized from templates linearized with BamHI. SP6 RNAs were microinjected into stage VI oocytes that were incubated in either the presence or absence of progesterone. $(B)$ Total RNA was extracted from stage VI and mature oocytes. $(C)$ RNA extracted from nonpolysomal (NP) and polysomal (P) fractions of stage VI and mature oocytes. RNAs were electrophoresed on a $1 \%$ agarose-formaldehyde gel. 
from a poly(A) removal activity that is bound to polysomes in mammalian cells (Brewer and Ross 1988).

The contribution of the poly $(A)$ tail to translational efficiency has been documented extensively for many eukaryotic mRNAs (for review, see Jackson and Standart 1990). The behavior of maternal mRNAs during Xenopus oocyte maturation indicates that the poly $(\mathrm{A})$ tail is a primary determinant of translational efficiency at this developmental stage. A wide variety of mRNAs that are translated efficiently in stage VI oocytes are released from polysomes upon their deadenylation at maturation (Sturgess et al. 1980; Dworkin and DworkinRastl 1985; Dworkin et al. 1985; Hyman and Wormington 1988; Wormington 1989). The core histone mRNAs are notable exceptions (Ruderman et al. 1979). There is increasing evidence that the poly(A) tail enhances translation initiation (Galili et al. 1988; Munroe and Jacobson 1990). Recent studies suggest that this enhancement is mediated through the poly $(\mathrm{A})$-binding protein (Sachs and Davis 1989, 1990). Thus, polyadenylated RNAs would be preferentially translated relative to deadenylated transcripts in mature oocytes.

Our results indicate that deadenylation results in the translational inactivation of $r$-protein mRNAs in mature oocytes. We have shown that insertion of the CPE, $(\mathrm{U})_{6} \mathrm{AU}$, into the 3' UTR of the r-protein Ll mRNA results in the retention of its poly(A) tail and its continued translation. We have not determined whether translation of the CPE-containing Ll-1095 mRNA is due to the presence of a poly $(A)$ tail or whether it requires the actual process of polyadenylation as does G10 mRNA (McGrew et al. 1989). The regulation of the two mRNAs may not be directly comparable. Although both the L1 and G10 mRNAs are polyadenylated to similar extents in stage VI oocytes, only the Ll mRNA is translated efficiently before maturation. Thus, the mechanism that maintains translation of the polyadenylated L1-1095 mRNA in mature oocytes may differ from the initial recruitment of G10 mRNA onto polysomes. It is important to note that the $(\mathrm{U})_{6} \mathrm{AU} \mathrm{CPE}$ alone cannot prevent the deadenylation and permit the translation of Ll-1095 RNA. If extraneous sequences located distal to the poly(A) tract are not removed from the L1-1095 RNA prior to microinjection, its poly $(A)$ tail is not retained and it is not associated with polysomes despite the presence of the CPE (S.M. Varnum, unpubl.). Similarly, McGrew et al (1989) showed that extraneous vector sequences prevented the $(\mathrm{U})_{6} \mathrm{AU}$-dependent polyadenylation and polysomal recruitment of G10 mRNA. These results do not rule out the possibility that the CPE functions as a translational enhancer in mature oocytes, but only in the context of polyadenylation.

The poly (A) tail is not a determinant of mRNA stability during maturation. Deadenylated maternal mRNAs are not degraded immediately in mature oocytes but are stably maintained as nonpolysomal transcripts (Dworkin and Dworkin-Rastl 1985; Dworkin et al. 1985; Hyman and Wormington 1988). It is likely, however, that deadenylation facilitates the eventual degradation of these maternal mRNAs after fertilization
(Baum and Wormington 1985; Duval et al. 1990).

The mechanism by which CPE-containing RNAs escape deadenylation remains to be defined. It is a formal possibility that all maternal mRNAs undergo poly(A) removal during maturation. Only mRNAs that contain a CPE would be substrates for a subsequent polyadenylation reaction. However, deadenylated intermediates of D7 or G10 mRNAs have not been detected during maturation (Fox et al. 1989; McGrew et al. 1989). Additional studies indicate that cytoplasmic polyadenylation precedes deadenylation. Cytoplasmic polyadenylation is an early event that precedes germinal vesicle breakdown (McGrew and Richter 1990). In contrast, deadenylation occurs after germinal vesicle breakdown and requires several hours for its completion (S.M. Varnum, unpubl.). It is possible that CPE-containing mRNAs may bind to factors that prevent deadenylation. An $82-\mathrm{kD}$ CPE-binding protein that is present in egg but not oocyte extracts is one candidate for such a factor (McGrew and Richter 1990). Wickens (1990) has suggested that the actual process or end product of polyadenylation may prevent deadenylation. For example, the poly $(\mathrm{A})$ tracts added to CPE-containing RNAs could contain modified adenylate residues that are resistant to deadenylation. Bass and Weintraub (1988) have identified an RNA helicase activity that also converts adenosine residues to inosine in Xenopus eggs, although its modification of $3^{\prime}$ poly $(\mathrm{A})$ tracts has not been addressed. Alternatively, the newly added poly $(A)$ tracts could bind to a different form of poly|A|-binding protein that prevents deadenylation.

A default mechanism of translational control is particularly applicable to oocyte maturation, given the enormous complexity of the maternal mRNA population (for review, see Davidson 1986). The absence of a specific sequence requirement for deadenylation provides a simple mechanism for the translational inactivation of unrelated mRNAs that are utilized during oogenesis but that otherwise share no common regulation. The dead enylation activity we have described is itself subject to regulation during maturation because poly $(\mathrm{A})$ removal does not occur until after germinal vesicle breakdown. It will also be of interest to determine how deadenylation activity is eliminated during embryogenesis, as the majority of new transcription commencing at the midblastula transition is due to the re-expression of sequences present previously as maternal mRNAs (Dworkin and Dawid 1980; Colot and Rosbash 1982; Baum and Worm ington 1985; Krieg et al. 1989). The development of an in vitro deadenylation system and purification of this RNA processing activity will address these aspects of its regulation and define its mechanism of poly(A) removal.

\section{Materials and methods}

Plasmid constructions and preparation of transcription templates

The plasmid pS22, isolated from a Xenopus stage 17 embryo cDNA library (Kintner and Melton 1987), contains a 531-bp S22 cDNA insert with a residual $3^{\prime}(\mathrm{A})_{-120}$ tail cloned downstream of the SP6 promoter in a derivative of pSP65 (Keiper and Wormington 1990). pS22 DNA was linearized with SacI, and 3'-pro- 
truding ends were removed with T4 DNA polymerase for use as a transcription template.

To construct the plasmid pS22-3'UTR, a fragment containing the S22 3' UTR was isolated by digestion of pS22 with NsiI and EcoRI. The fragment was cloned into the PstI and EcoRI sites of pSP65AT (Baum et al. 1988). pS22-3'UTR DNA was linearized with EcoRI for use as a transcription template.

To construct plasmids encoding mutant S22-3'UTR RNAs or the G52 RNA derived from the $3^{\prime}$ UTR of $\beta$-globin mRNA, oligonucleotides specific for each sequence were synthesized. Complementary oligonucleotides containing a $5^{\prime}$ BgIII site and a $3^{\prime}$ Sacl site were phosphorylated, annealed by heating to $75^{\circ} \mathrm{C}$, and cooled slowly to room temperature. The annealed oligonucleotides were ligated into the BgIII and SacI sites of pSP65$\mathrm{L1A}^{+}$(Baum et al. 1988). The oligonucleotide inserts containing a $\left[(\mathrm{dA}) \cdot(\mathrm{dT}) \|_{100}\right.$ tract were subsequently isolated by digestion with BglII and BamHI and ligated into the BamHI site of pSP65. The plasmid pS22-mut 2 contained the oligonucleotide sequence 5'-GATCTATAAATGACACGCTGCAGACCCGGGAGAAAGTAATGTGGATTGTTGAGCT annealed to its complement. The plasmid pS22-mut3 contained the oligonucleotide sequence 5'-GATCTATAAATGACACTTCTGTTTAATAAACCTGCAGCCAACATCAGGCAGAGCT annealed to its complement. The plasmid pG52 contained the oligonucleotide sequence 5'-GATCTCTAATCGTATCTGCTCCTAATAAAAAGAAAGTTTCTTCACATTCTGAGCT annealed to its complement. pS22-mut2, pS22-mut3, and pG52 were linearized with $\mathrm{BamHI}$ for use as transcription templates.

To construct pHP1, the oligonucleotide sequence 5 -CCATGCCCCCCCCCCCCCCCCCCCCCCCCTAAGAGCT and its complement were phosphorylated and annealed as above and inserted into the SacI site of pSP65AT. pHPl DNA was linearized with $B a m H I$ for use as a transcription template.

$\mathrm{pLlA}^{+}$contains the Ll cDNA inserted at the EcoRI site of pSP65AT (Baum et al. 1988). To construct pL1-1095, the sequence $5^{\prime}$-TTTTTTAT was inserted by site-directed mutagenesis (Olsen and Eckstein 1990) 8 bp upstream of the poly|A) addition site AATAAA in pLlA ${ }^{+}$. pLl-20 and pL1-42 were derived from deletion mutants of $\mathrm{pLlA}^{+}$in which the $[(\mathrm{dA}) \cdot(\mathrm{dT})]_{100}$ tract had been reduced to either 20 or $42 \mathrm{bp}$, respectively. pLlA ${ }^{+}, \mathrm{pLl}-1095, \mathrm{pLl}-20$, and pLl-42 DNAs were linearized with BamHI for use as transcription templates.

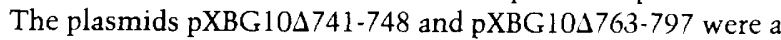
gift from Lynn McGrew and Joel Richter (McGrew et al. 1989). Both DNAs were linearized with SalI for use as transcription templates.

pSP64-X $\beta m$ contains a Xenopus $\beta$-globin cDNA cloned into pSP64 (Krieg and Melton 1984). pSP64-Xßm was linearized with PstI, and 3 '-protruding ends were removed with T4 DNA polymerase for use as a transcription template.

DNA sequencing was performed on double-stranded plasmid DNA by the dideoxy chain-termination method (Sanger et al. 1977) using a Sequenase sequencing kit (U.S. Biochemical Corp.).

\section{In vitro transcription and oocyte microinjection}

Transcripts were synthesized in vitro with SP6 RNA polymerase according to Krieg and Melton (1984) except that reactions contained $1 \mathrm{mM} \mathrm{m}{ }^{7} \mathrm{GpppG}, 25 \mu \mathrm{M}$ GTP, $25 \mu \mathrm{M}$ UTP, and $50 \mu \mathrm{Ci}$ of $\left[\alpha^{-32} \mathrm{P}\right] \mathrm{UTP}(\sim 800 \mathrm{Ci} / \mathrm{mmole}$, New England Nuclear). Reactions were incubated for $1-2 \mathrm{hr}$ at $37^{\circ} \mathrm{C}$. RNAs were extracted with phenol/chloroform and precipitated twice with ethanol to remove unincorporated nucleotides. The $3^{\prime}$ termini

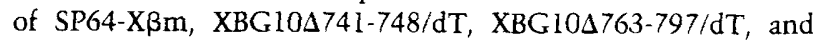
L1-1095 RNAs were removed by digestion with RNase $\mathrm{H}$ after hybridization to oligoldT $\left.\right|_{12-18}$ as described (Hyman and Wormington 19881 . Poly $(\mathrm{A})$ tails between 50 and 100 adenylate residues in length were added to SP64-X $\beta \mathrm{m}$ and XBG10 $0741-748$ / $\mathrm{dT} / \mathrm{A}^{+}$RNAs with $E$. coli poly(A) polymerase as described (McGrew et al. 1989). Ethanol-precipitated RNAs were rinsed twice with $70 \%$ ethanol and dissolved in $88 \mathrm{~mm} \mathrm{NaCl}$ prior to microinjection.

Two days before oocyte isolation, Xenopus females were primed with 25-50 units of pregnant mare serum (Sigma). Fully grown stage VI oocytes were isolated from ovarian fragments, defolliculated with collagenase, and maintained at $18^{\circ} \mathrm{C}$ in modified Barth's saline (Gurdon 1976). Oocytes were injected with $20-40 \mathrm{nl}$ of solution containing $\sim 0.2-1.0 \mathrm{ng}$ of RNA, which is nearly equivalent to the endogenous levels of individual r-protein mRNAs (Davidson 1986). Maturation was induced by the addition of progesterone to a final concentration of $1 \mu \mathrm{g} / \mathrm{ml}$. Mature oocytes were collected 5-8 hr after the appearance of a white spot at the animal pole that was indicative of germinal vesicle breakdown.

\section{RNA isolation and analysis}

Total RNA was extracted from microinjected oocytes as de scribed (Wormington 1986). In some cases, total RNA prepara tions were hybridized to oligo $(\mathrm{dT})_{12-18}$ and deadenylated in vitro with RNase $\mathrm{H}$, as described by Hyman and Wormington (1988), except that reactions were incubated for $1 \mathrm{hr}$. Polysomal and nonpolysomal RNAs were isolated from cytoplasmic extracts centrifuged through $20 \%$ sucrose cushions as described in detail (Baum et al. 1988). RNAs were resolved by electrophoresis in either $1 \%$ or $1.3 \%$ agarose $-2.2 \mathrm{M}$ formaldehyde gels or in $4 \%$ or $6 \%$ polyacrylamide--7 $\mathrm{M}$ urea gels (Maniatis et al. 19821. Each lane contained the RNA equivalent of one to two oocytes. Gels were dried, and the radiolabeled RNAs were detected by autoradiography. Differences in the intensities of RNA bands between lanes are due to variability in the stability of the injected RNAs in stage VI or mature oocytes.

\section{Acknowledgments}

We are grateful to Joel Richter and Lynn McGrew for generously providing G10 plasmids and we thank Marv Wickens for communicating results prior to publication. We also thank Linda Hyman, Brett Keiper, Lynn McGrew, Joel Richter, Michael Rosbash, and Ranjan Sen for many stimulating discussions. This work was supported by National Institutes of Health grant HD17691 to W.M.W.

The publication costs of this article were defrayed in part by payment of page charges. This article must therefore be hereby marked "advertisement" in accordance with 18 USC section 1734 solely to indicate this fact.

\section{References}

Bass, B.L. and H. Weintraub. 1988. An unwinding activity that covalently modifies its double-stranded RNA substrate. Cell 55: $1089-1098$.

Baum, E. Z. and W.M. Wormington. 1985. Coordinate expression of ribosomal protein genes during Xenopus development. Dev. Biol. 111: 488-498.

Baum, E. Z., L.E. Hyman, and W.M. Wormington. 1988. Posttranslational control of ribosomal protein $\mathrm{Ll}$ accumulation in Xenopus oocytes. Dev. Biol. 126: 141-149.

Brewer, G. and J. Ross. 1988. Poly|A| shortening and degradation of the 3' A + U-rich sequences of human c-myc mRNA in a cell-free system. Mol. Cell. Biol. 8: 1697-1708.

Cleveland, D.W. 1988. Autoregulated instability of tubulin 
mRNAs: A novel eukaryotic regulatory mechanism. Trends Biochem. 13: 339-343.

Colot, H.V. and M. Rosbash. 1982. Behavior of individual maternal pA + RNAs during embryogenesis of Xenopus laevis. Dev. Biol. 94: 79-86.

Davidson, E. H. 1986. Gene activity in early development. Academic Press, New York.

Duval, C., P. Bouvet, F. Omilli, C. Roghi, C. Dorel, R. Leguellec, J. Paris, and H.B. Osborne. 1990. Stability of maternal mRNA in Xenopus embryos: Role of transcription and translation. Mol. Cell. Biol. 10: 4123-4129.

Dworkin, M.B. and I.B. Dawid. 1980. Use of a cloned library for the study of abundant poly $\mid \mathrm{A})^{+}$RNA during Xenopus laevis development. Dev. Biol. 76: 449-464.

Dworkin, M.B. and E. Dworkin-Rastl. 1985. Changes in RNA titers and polyadenylation during oogenesis and oocyte maturation in Xenopus laevis. Proc. Natl. Acad. Sci. 82: $7636-$ 7640.

Dworkin, M.B., A. Shrutkowski, and E. Dworkin-Rastl. 1985. Mobilization of specific maternal RNA species into polysomes after fertilization in Xenopus laevis. Proc. Natl. Acad. Sci. 82: 7636-7640.

Fox, C.A., M.D. Sheets, and M.P. Wickens. 1989. Poly $(A)$ addition during maturation of frog oocytes: Distinct nuclear and cytoplasmic activities and regulation by the sequence UUUUUAU. Genes Dev. 3: 2151-2162.

Galili, G., E.E. Kawata, L.D. Smith, and B.A. Larkins. 1988. Role of the $3^{\prime}$-poly(A) sequence in translational regulation of mRNAs in Xenopus laevis oocytes. I. Biol. Chem. 263: $5764-5770$.

Gurdon, J.B. 1976. Injected nuclei in frog oocytes: Fate, enlargement and chromatin dispersal. I. Embryol. Exp. Morphol. 36: $523-540$.

Hyman, L.E. and W.M. Wormington. 1988. Translational inactivation of ribosomal protein mRNAs during Xenopus oocyte maturation. Genes Dev. 2: 598-605.

Jackson, R.J. and N. Standart. 1990. Do the poly(A) tail and 3' untranslated region control mRNA translation? Cell 62: $15-24$.

Keiper, B.D., and W.M. Wormington. 1990. Nucleotide sequence and $40 \mathrm{~S}$ subunit assembly of Xenopus laevis ribosomal protein S22. I. Biol. Chem. 265: 19397-19400.

Kintner, C.R. and D.A. Melton. 1987. Expression of Xenopus N-CAM RNA in ectoderm is an early response to neural induction. Development 99: 311-325.

Krieg, P.A. and D.A. Melton. 1984. Functional messenger RNAs are produced by SP6 in vitro transcription of cloned cDNAs. Nucleic Acids Res. 12: 7057-7070.

Krieg, P.A., S.M. Varnum, W.M. Wormington, and D.A. Melton. 1989. The mRNA encoding elongation factor $1-\alpha$ $(E F-1 \alpha)$ is a major transcript at the midblastula transition in Xenopus. Dev. Biol. 133: 93-100.

Lee, G., R. Hynes, and M. Kirschner. 1984. Temporal and spatial regulation of fibronectin in early Xenopus development. Cell 36: 729-740.

Maniatis, T., E.F. Fritsch, and J. Sambrook. 1982. Molecular cloning: A laboratory manual. Cold Spring Harbor Laboratory, Cold Spring Harbor, New York.

Mariottini, P., C. Bagni, F. Annesi, and F. Amaldi. 1988. Isolation and nucleotide sequences of cDNAs for Xenopus laevis ribosomal protein S8: Similarities in the $5^{\prime}$ and $3^{\prime}$ untranslated regions of mRNAs for various r-proteins. Gene 67: 6974.

Marzluff, W.F. and N.B. Pandey. 1988. Multiple regulatory steps control histone mRNA concentrations. Trends Biochem. 13: 49-52.
McGrew, L.L. and J.D. Richter. 1990. Translational control by cytoplasmic polyadenylation during Xenopus oocyte maturation: Characterization of cis and trans elements and regulation by cyclin/MPF. EMBO I. 9: 3743-3751.

McGrew, L.L., E. Dworkin-Rastl, M.B. Dworkin, and I.D. Richter. 1989. Poly(A) elongation during Xenopus oocyte maturation is required for translational recruitment and is mediated by a short sequence element. Genes Dev. 3: 803815.

Munroe, D. and A. Jacobson. 1990. mRNA poly(A) tail: A 3' enhancer of translational initiation. Mol. Cell. Biol. 10: $3441-3455$.

Olsen, D.B. and F. Eckstein. 1990. High-efficiency oligonucleotide-directed plasmid mutagenesis. Proc. Natl. Acad. Sci. 87: 1451-1455.

Paris, J. and J.D. Richter. 1990. Maturation-specific polyadenylation and translational control: Diversity of cytoplasmic polyadenylation elements, influence of poly (A) tail size, and formation of stable polyadenylation complexes. Mol. Cell. Biol. 10: $5634-5645$.

Richter, J.D., W.J. Wasserman, and L.D. Smith. 1982. The mechanism for increased protein synthesis during Xenopus oocyte maturation. Dev. Biol. 89: 159-167.

Ruderman, J.V., H.R. Woodland, and E.A. Sturgess. 1979. Modulations of histone messenger RNA during the early development of Xenopus laevis. Dev. Biol. 71: 71-82.

Sachs, A.B. and R.W. Davis. 1989. The poly|A| binding protein is required for poly(A) shortening and $60 \mathrm{~S}$ ribosomal subunit-dependent translation initiation. Cell 58: 857-867.

- 1990. Translation initiation and ribosomal biogenesis: Involvement of a putative rRNA helicase and RPL46. Science 247: 1077-1079.

Sagata, N., K. Shiokawa, and K. Yamana. 1980. A study on the steady-state population of poly $(A)^{+}$RNA during the early development of Xenopus laevis. Dev. Biol. 77: 431-448.

Sagata, N., M. Oskarsson, T. Copeland, J. Brumbaugh, and G.F. Vande Woude. 1988. Function of c-mos proto-oncogene product in meiotic maturation in Xenopus oocytes. Nature 335: $519-525$.

Sanger, F., S. Nicklen, and A.R. Coulson. 1977. DNA sequencing with chain-terminating inhibitors. Proc. Natl. Acad. Sci. 74: 5463-5467.

Stick, R. and P. Hausen. 1985. Changes in the nuclear lamina composition during early development of Xenopus laevis. Cell 41: 191-200.

Sturgess, E.A., J.E.M. Ballantine, H.R. Woodland, T.J. Mohun, C.D. Lane, and G.J. Dimitriadis. 1980. Actin synthesis during the early development of Xenopus laevis. J. Embryol. Exp. Morphol. 58: 303-339.

Taylor, M.V., M. Gusse, G.I. Evan, N. Dathan, and M. Mechali. 1986. Xenopus myc proto-oncogene during development: Expression as a stable maternal mRNA uncoupled from cell division. EMBO I. 5: 3563-3570.

Wickens, M. 1990. In the beginning is the end: Regulation of poly $(\mathrm{A})$ addition and removal during early development. Trends Biochem. 15: 320-324.

Woodland, H.R., J.M. Flynn, and A.J. Wyllie. 1979. Utilization of stored mRNA in Xenopus embryos and its replacement by newly synthesized transcripts: Histone $\mathrm{Hl}$ synthesis using interspecies hybrids. Cell 18: 165-171.

Wormington, W.M. 1986. Stable repression of ribosomal protein Ll synthesis in Xenopus oocytes by microinjection of antisense RNA. Proc. Natl. Acad. Sci. 83: 8639-8643.

1989. Developmental expression and 5S rRNA-binding activity of Xenopus laevis ribosomal protein L5. Mol. Cell Biol. 9: 5281-5288. 


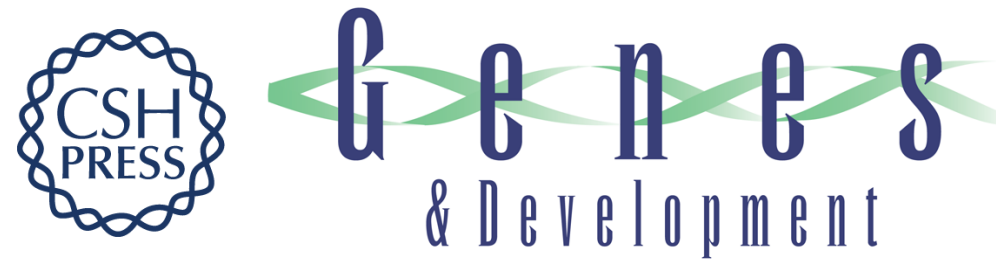

\section{Deadenylation of maternal mRNAs during Xenopus oocyte maturation does not require specific cis-sequences: a default mechanism for translational control.}

S M Varnum and W M Wormington

Genes Dev. 1990, 4:

Access the most recent version at doi:10.1101/gad.4.12b.2278

References This article cites 41 articles, 17 of which can be accessed free at:

http://genesdev.cshlp.org/content/4/12b/2278.full.html\#ref-list-1

License

Email Alerting

Service

Receive free email alerts when new articles cite this article - sign up in the box at the top right corner of the article or click here.

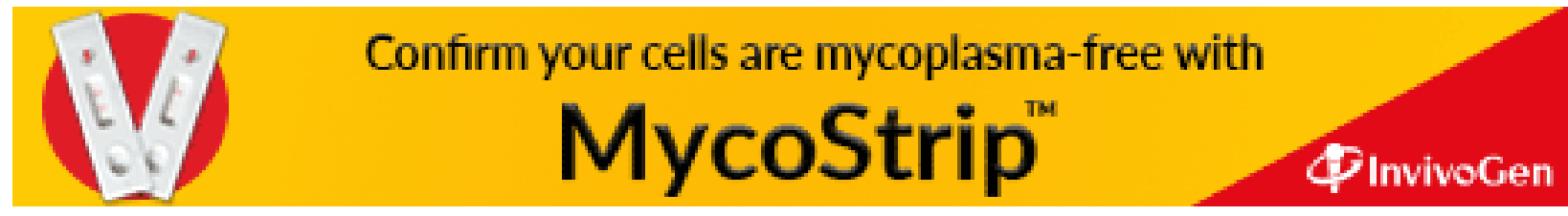

